\title{
Oscillating decorated interfaces in parametrically driven systems
}

\author{
E. Berrios-Caro, ${ }^{1}$ M. G. Clerc, ${ }^{1}$ M. A. Ferre, ${ }^{1}$ and A. O. Leon ${ }^{2}$ \\ ${ }^{1}$ Departamento de Física, Facultad de Ciencias Físicas y Matemáticas, Universidad de Chile, Casilla 487-3, Santiago, Chile \\ ${ }^{2}$ Institute for Materials Research, Tohoku University, Sendai 980-8577, Japan
}

(Received 25 September 2017; published 16 January 2018)

\begin{abstract}
Macroscopic systems forced by the temporal modulation of their parameters exhibit complex interfaces between symmetric states. Here we investigate the origin of the transition from a flat to an oscillating decorated interface. Based on a model that describes a magnetic plane under the influence of an oscillating magnetic field and an extended Josephson junction under the influence of an alternating current, we derive a simple model that accounts for the interface dynamics. Analytically this model allows us to reveal that this transition is a parametric resonance between the frequencies of interface modes and the forcing. Numerical simulations of magnetic systems, extended Josephson junctions, and our simplified model show quite good agreement.
\end{abstract}

DOI: 10.1103/PhysRevE.97.012207

\section{INTRODUCTION}

Macroscopic systems under the influence of injection and dissipation of energy usually exhibit coexistence of different states [1-3]. As results of the inherent fluctuations of macroscopic systems, they are characterized by exhibiting spatial domains with different equilibria. These domains are separated by interfaces or domain walls or fronts [3]. Depending on the relative stability of the equilibria, these interfaces usually move in a way to minimize the free energy of the system under study and give rise to a rich spatiotemporal dynamics [4]. This phenomenon is known as front propagation. However, the previous scenario changes when domain walls separate two symmetric states. This type of interface is commonly called a kink [5]. The propagation mechanism of these domains is mediated by the curvature of the interface. This propagation phenomenon is known as the Gibbs-Thomson effect [2], which usually leads to a flat and motionless stable interface. In the case of nonvariational systems, when they present a spontaneous breaking of symmetry, the front between symmetric states becomes propagative [6-8]. The velocity of the interface is determined by the symmetry-breaking parameter and its curvature. The above phenomenon is well known as the nonvariational Ising-Bloch transition [6]. Interfaces that connect symmetric states are generically observed in parametrically driven systems, namely, systems forced by mean of temporal modulation of their parameters. These interfaces exist because the parametric forcing generates oscillatory solutions, which may be in phase or out of phase with respect to the forcing. In a large range of parameters, these interfaces are motionless and flat domain walls. However, by changing the parameters these interfaces can undergo transverse instabilities, which can cause the emergence of spatial structures along them. In the context of vertically driven granular media this type of interface has been observed and is called a decorated interface $[9,10]$. Likewise, magnetic systems subjected to external magnetic fields are characterized by the display of domains walls between symmetrical magnetic states [11]. Numerical simulations of a magnetic plane subjected to an oscillatory magnetic field show that this system can also exhibit oscillating decorated interfaces, as illustrated in Fig. 1(a). Hence, the transition from flat to decorated interfaces is a robust phenomenon of parametrically driven systems.

\section{DECORATED INTERFACES}

The purpose of this article is to understand the transition from flat to oscillating decorate interfaces in parametrically forced systems. Numerical simulations of a magnetic plane with a strong anisotropy driven by an oscillating magnetic field show a transition from a flat to a decorated interface. Based on the continuous description of the magnetic plane, i.e., the Landau-Lifshitz-Gilbert equation [12], we derive the parametrically driven sine-Gordon equation for the azimuthal angle of magnetization. This model also describes the dynamics of the phase difference of an extended Josephson junction under the influence of an alternating current [13]. A schematic representation of this device is shown in Fig. 1(b). Numerical simulations of the parametrically driven sine-Gordon model also exhibit a transition from a flat to an oscillating decorated interface [see the inset in Fig. 1(b)]. In the conservative limit, this model has an analytic solution, the $2 \pi$ kink. By using the inverse scattering method and perturbative theory, we derive a simple model that accounts for the dynamics of the interface. This model corresponds to a parametrically forced wave equation. This model allows us to reveal analytically that this transition is a parametric resonance between the frequencies of interface modes and of the forcing. Numerical simulations of magnetic systems, extended Josephson junctions, and our simplified model show quite fair agreement.

Let us consider a ferromagnetic plane under the influence of an oscillatory magnetic field, as illustrated in Fig. 1(a). Ferromagnetic films are described by a magnetization $\mathbf{M}(T, \mathbf{R})$, which can vary over time $T$ and along the in-plane coordinates $\mathbf{R}$. Introducing the dimensionless quantities, $\mathbf{m}=\mathbf{M} / M_{s}, t=$ $T / \tau$, and $\mathbf{r}=\mathbf{R} / l_{\mathrm{ex}}$, where $\left\{M_{s}, l_{\mathrm{ex}}, \tau\right\}$ are the magnetization norm, the exchange length, and the characteristic time scale, respectively. For example, for permalloy $M_{s}=800 \mathrm{kA} / \mathrm{m}$, $l_{\mathrm{ex}}=5.7 \mathrm{~nm}$, and $\tau=6 \mathrm{ps}$. The magnetization dynamics obeys (the dimensionless Landau-Lifshitz-Gilbert equation [12])

$$
\partial_{t} \mathbf{m}=-\mathbf{m} \times\left[h \mathbf{e}_{x}-\beta m_{z} \mathbf{e}_{z}+\nabla^{2} \mathbf{m}-\alpha \partial_{t} \mathbf{m}\right],
$$


(a)
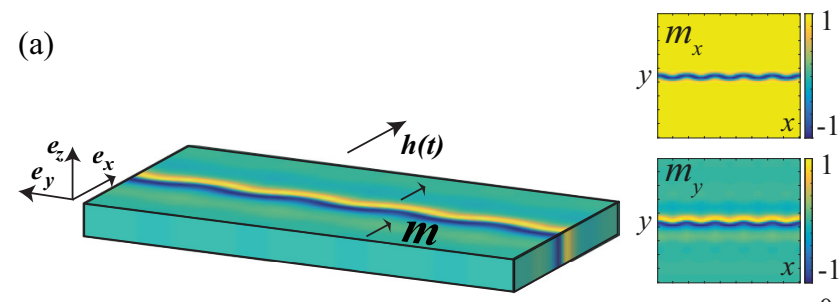

(b)
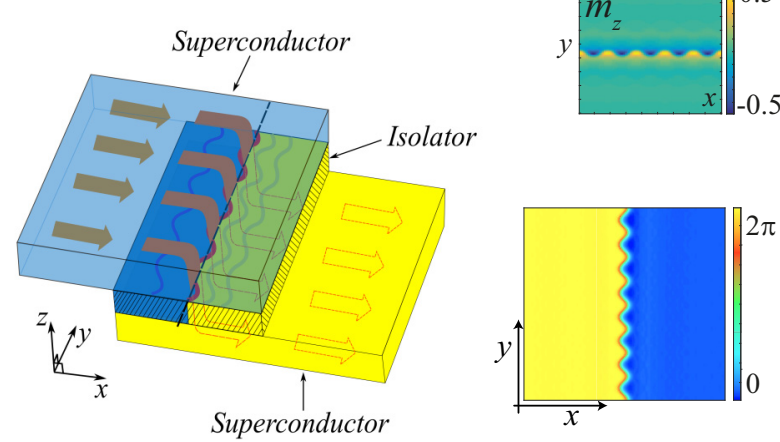

FIG. 1. Decorated interfaces in parametrically driven systems. (a) Domain wall in a parametrically driven magnetic plane obtained from numerical simulation of Eq. (1) with $h(t)=H_{0}+h_{0} \cos (\omega t)$, $H_{0}=1, h_{0}=0.4, \omega=2.94, \beta=6$, and $\alpha=0.1$. (b) Schematic representation of an extended Josephson junction, which is composed of two superconductors separated by an insulating strip under the influence of an alternating superconducting current. The inset shows a numerical simulation of Eq. (2) with $\omega_{0}=1, \gamma=0.4, \omega=1.6$, $\beta=1$, and $\mu=0.1$.

where $\left\{\mathbf{e}_{x}, \mathbf{e}_{y}, \mathbf{e}_{z}\right\}$ are the unit vectors along the respective Cartesian axes in the magnetization space and $h$ is the dimensionless intensity of the external magnetic field, which is along the $\mathbf{e}_{x}$ axis. For $h>0$, the stable equilibrium of the system is the magnetization pointing along the magnetic field $\mathbf{m}=\mathbf{e}_{x}$. The parameter $\beta>0$ accounts for the anisotropy of the film and it favors configurations where the magnetization lies on the $x y$ plane. The Laplacian of the magnetization $\nabla^{2} \mathbf{m} \equiv\left(\partial_{x x}+\partial_{y y}\right) \mathbf{m}$ stands for the ferromagnetic exchange interaction and it favors smooth magnetic states. The term proportional to $\alpha$ is a Rayleigh-like dissipation function known as Gilbert damping [12] and it accounts for energy dissipation. Typical values are in the range $10^{-3} \leqslant \alpha \leqslant 5 \times 10^{-2}$ and can be enhanced significantly by doping the film with heavy magnetic atoms. Numerical simulations of this system with an external oscillatory magnetic field $h(t)=H_{0}-h_{0} \sin (\omega t)$ exhibit oscillating decorated interfaces [cf. Fig. 1(a)]. To describe this phenomenon, one can introduce a spherical representation of the magnetization vector $\mathbf{m}=\sin (\theta)\left[\cos (\phi) \mathbf{e}_{x}+\right.$ $\left.\sin (\phi) \mathbf{e}_{y}\right]+\cos (\theta) \mathbf{e}_{z}$, where $\theta(t, \mathbf{r})$ and $\phi(t, \mathbf{r})$ are, respectively, the polar and azimuthal angles. In the limit of large magnetic anisotropy, most of the magnetization vector lies in the $x y$ plane and the external magnetic field acts in the same manner as gravity for coupled mechanical oscillators [14,15]. Considering the scaling $|\theta-\pi / 2| \sim \alpha \sim h \sim \nabla^{2} \phi \sim 1 / \beta \ll 1$ and $\phi \sim \partial_{t} \phi \sim 1$, one gets at leading order that $\theta \approx \pi / 2+\partial_{t} \phi / \beta$ and $\phi$ satisfies (the parametrically driven damped sine-Gordon model)

$$
\partial_{t t} \phi+\omega_{0}^{2} \sin (\phi)+\beta \nabla^{2} \phi=\gamma \sin (\omega t) \sin (\phi)-\mu \partial_{t} \phi,
$$

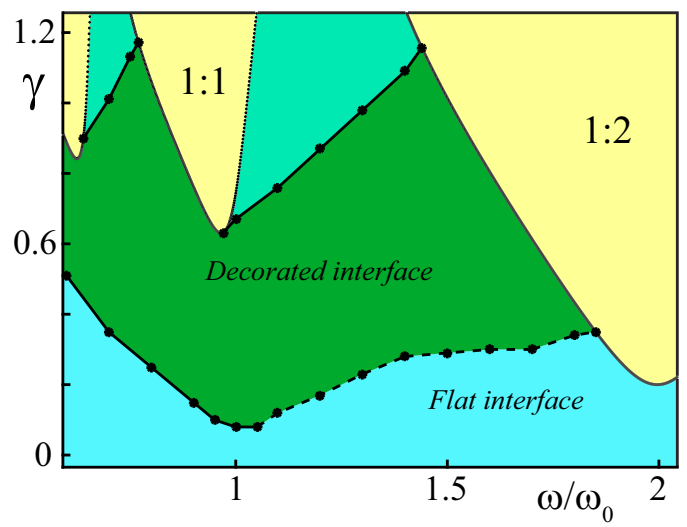

FIG. 2. Stability diagram of the domain walls or $2 \pi$ kinks for the sine-Gordon model (2) for $\mu=0.1$ and $\beta=1$. The horizontal and vertical axes represent, respectively, the frequency and amplitude of the forcing. Regions 1:2 and 1:1 account for the Arnold tongues (parametric resonances). The dashed (solid) bottom curve represents the supercritical (subcritical) transition from a flat to an oscillating decorated interface.

where $\omega_{0} \equiv \sqrt{H_{0} \beta}$ is the natural frequency, $\gamma \equiv h_{0} \beta$ is the amplitude of the parametrically forcing, and $\mu=\alpha \beta$ is the dissipation coefficient. This model (2) accounts also for the dynamics of the phase difference of an extended Josephson junction under the influence of an alternating current $[13,16]$. Notice that Eq. (1) is invariant under the renormalization $(h, \beta, t, \mathbf{r}) \rightarrow\left(h \beta^{-1}, 1, \beta t, \beta^{1 / 2} \mathbf{r}\right)$ and then the same solutions as for strong-anisotropy films can persist in systems with moderate anisotropy. For materials such as permalloy, we have $\beta \equiv 1$ and the sine-Gordon model is obtained from Eq. (1) using the scaling $|\theta-\pi / 2|^{2} \sim \alpha^{2} \sim h \sim \nabla^{2} \phi \sim \partial_{t t} \phi \sim \epsilon \ll$ 1 , where $\epsilon$ is a small expansion parameter. Thus, without loss of generality, we can consider the temporal and spatial units in the natural frequency and the anisotropy length, so one can choose $\omega_{0}=\beta=1$ in Eq. (2).

The model (2) has domain walls connecting the zero to the $2 \pi$ state. As a result of parametric forcing, these kinks are characterized by emitting evanescent nonlinear waves [15]. For the two-dimensional systems, one expects these domain walls to be flat. Figure 2 shows the bifurcation diagram of $2 \pi$-kink solutions. For small forcing amplitudes, one finds that these interfaces are flat, namely, if one disturbs the flat interface it evolves so that it is flattened again. However, as the forcing amplitude is increased, these interfaces exhibit a transverse instability that results in oscillating decorated interfaces. Figure 3(a) depicts an oscillating decorated interface and its spatiotemporal evolution. Indeed, the interface oscillates with the forcing frequency. Numerically, we can extract the interface position $P(y, t)$, which is a one-dimensional scalar field. This field allows us to characterize the interface dynamics [see the right panel of Fig. 3(a)]. Depending on the frequency, the transition can be of supercritical or subcritical type (cf. Fig. 2).

\section{UNIVERSALITY OF DECORATED INTERFACES}

To understand the rich interface dynamics, we consider the nearly integrable limit, that is, $\mu \sim \gamma \ll 1$. Then the terms on 

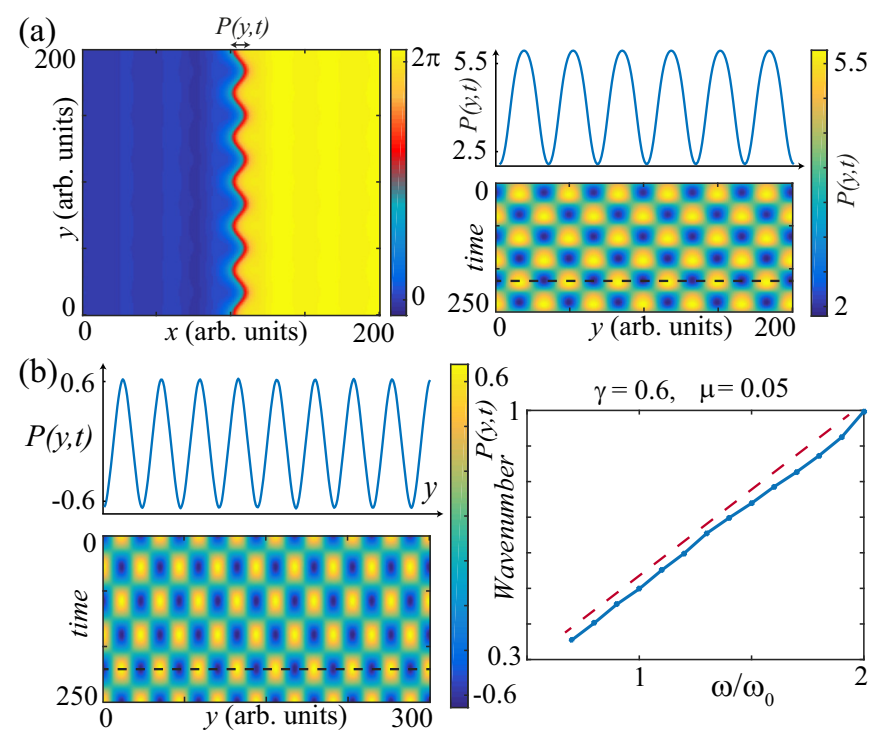

FIG. 3. Spatiotemporal dynamics of decorated interfaces. (a) Decorated interface at a given time and spatiotemporal diagram of the interface position $P(y, t)$ for the sine-Gordon model (2) for $\omega_{0}=1$, $\gamma=0.4, \omega=1.2, \beta=1$, and $\mu=0.1$. (b) Left panel shows the profile and spatiotemporal diagram of the interface position $P(y, t)$ of Eq. (6) for $\mu=0.05, \gamma=0.3$, and $\omega=1.8$. Right panel illustrates the relation between the interface wave number and the forcing frequency.

the right-hand side of Eq. (2) are of perturbative nature. In the conservative limit, the sine-Gordon model has a family of analytical propagative solutions for $2 \pi$ kinks. We consider the ansatz for a two-dimensional $2 \pi \mathrm{kink}$

$$
\phi(x, y, t)=4 \arctan \left[\exp \left(-\frac{x-P(y, t)}{\sqrt{1-v(y, t)^{2}}}\right),\right.
$$

where $P=P(y, t)$ and $v(y, t)$ are, respectively, the position and the speed of the interface. Using the inverse scattering transform and perturbative theory [17], one obtains the set of equations

$$
\begin{aligned}
\partial_{t} v=-\mu v\left(1-v^{2}\right)+\left(1-v^{2}\right) \partial_{y y} P+v \partial_{y} v \partial_{y} P & \\
\partial_{t} P=v & -\frac{\gamma}{2} v\left(1-v^{2}\right) \sin \omega t \\
+ & \frac{v}{2}\left[\left(\partial_{y} P\right)^{2}-\frac{\pi^{2}}{6}\left[v \partial_{y y} v+\left(\partial_{y} v\right)^{2}\right]\right] .
\end{aligned}
$$

In the conservative $(\mu=\gamma=0)$ and uniform $\left(\partial_{y} P=\partial_{y} v=\right.$ 0 ) limits, Eq. (5) reduces to the relation $v=\partial_{t} P$. To compute the corrections to this limit, we apply the temporal derivative of Eq. (5), use Eq. (4), and consider only the dominant order terms

$$
\begin{aligned}
\partial_{t t} P= & \partial_{y y} P-\mu \partial_{t} P-\frac{\gamma \omega}{2} \partial_{t} P \cos \omega t+\partial_{t} P \partial_{y} P \partial_{t y} P \\
& -\frac{\gamma}{2}\left(\mu \partial_{t} P+\partial_{y y} P\right) \sin \omega t+\left(\partial_{y y} P-\mu \partial_{t} P\right)\left(\partial_{y} P\right)^{2} .
\end{aligned}
$$

Hence, the interface position satisfies a parametrically driven damped nonlinear wave equation. In the case that the forcing is ignored $(\gamma=0)$, Eq. (6) corresponds to a dissipative wave equation, which exhibits a flat interface as a unique equilibrium. When increasing the forcing amplitude $\gamma$, the flat state becomes unstable showing an oscillatory solution. Hence, this reduced model for the interface position accounts for the observed dynamics (cf. Fig. 2). As $\gamma$ increases, the system exhibits a parametric resonance [18]. Note that wave equation (6) does not have an intrinsic natural frequency. However, each linear mode of wave number $k$ has a frequency $k$. Therefore, the system can exhibit a resonance when the forcing frequency satisfies $\omega \sim \pm k / 2$ [18] [see Fig. 3(b)]. This resonance is responsible for the transition from a flat to an oscillating decorated interface. Figure 3(b) shows the profile and the spatiotemporal diagram of interface position $P(y, t)$ when the model (6) has a parametric resonance. The right panel of this figure illustrates the relation between the wave number and the forcing frequency. Note that the wave number increases with forcing frequency. This function, in general, is not linear, which shows that to give a better account of the resonance one must include higher dispersive terms (such as $\partial_{y y y y} P$ ) in the nonlinear wave equation (6). Since the amplitudes of the decorated interface are more commonly found near the natural frequency $\omega_{0}$ and the linear condition for parametric instability is valid near this region, our approximation of the wave equation (6) will be valid in this region.

To study this parametric resonance analytically close to natural frequency $\omega_{0}$, we consider the slowly varying envelope method [19]. Let us consider the ansatz $P(y, t)=$ $A(t) \cos \left(k_{n} y\right) e^{i \omega t}+w(A, y, t)+$ c.c., where $A$ is the envelope of standing wave, $w$ is a small correction function, c.c. denotes the complex conjugate, and $\omega=2 k_{n}+v$, with $v$ the detuning parameter between the frequencies of interface modes and of the forcing. The wave number $k_{n}=2 \pi n / L$ is compatible with boundary conditions, $n=\{1,2,3, \ldots\}$, and $L$ is the system width. Introducing this ansatz into Eq. (6), linearizing in $w$, and imposing the solvability conditions, one gets

$$
\partial_{t} A=-i \nu A-\frac{\mu}{2} A+\frac{\gamma}{8}\left(2 k_{n}+v\right) \bar{A}-\frac{i}{4} k^{3} A|A|^{2} .
$$

This equation has a trivial equilibrium $A=0$, which corresponds to a flat quiescent interface. This state becomes unstable through a stationary instability at

$$
\Gamma_{n} \equiv \gamma^{2}\left(2 k_{n}+v\right)^{2} / 64-v^{2}-\mu^{2} / 4=0,
$$

which is a subcritical and a supercritical bifurcation for negative and positive detuning, respectively. These curves, in the context of parametric instabilities, are often called Arnold tongues. Note that the constraint $\omega \approx \omega_{0}$ sets a maximum $n$ index for our approximation. Figure 4 shows the instability curve $\Gamma_{n}$ in the frequency and the amplitude of the forcing space. These parametric instabilities give rise to the equilibria $A=R e^{i \phi}$, where $\cos (2 \phi)=4 \mu / \gamma\left(2 k_{n}+v\right) 9$ and $R=\sqrt{\left[-v \pm \sqrt{\gamma^{2}\left(2 k_{n}+v\right)^{2} / 4-4 \mu^{2}}\right] / k_{n}^{3}}$. These solutions account for decorated interfaces, which correspond to standing waves for the interface of frequency $\omega$ and amplitude $R$, as illustrated in Fig. 3. From the formula of $R$, one can infer that the decorated interfaces appear or disappear by a saddle-node bifurcation at the curve $\Phi_{0} \equiv \gamma^{2} \omega^{2} / 4-4 \mu^{2}$. Hence, in the region in the space of parameters between the $\Gamma_{0}$ curve and the last stable Arnold tongue, we observe 


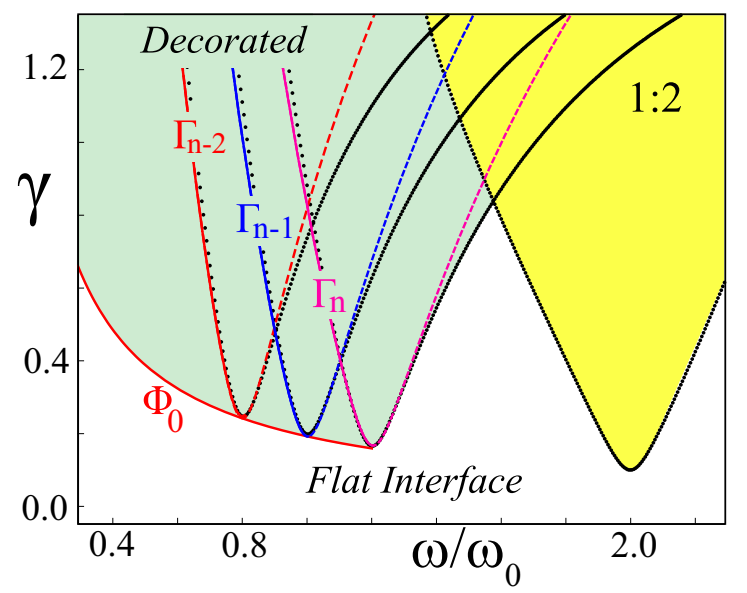

FIG. 4. Bifurcation diagram of the amplitude equation (7). The $\Gamma_{k}$ represent the Arnold tongues, whereas the solid and dashed curves represent the numerical and analytical tongues, respectively. The solid and dashed curves stand for the subcritical and supercritical bifurcations of the flat interface, respectively. The bottom solid curve $\Phi_{0}$ represents the saddle-node bifurcation of the decorated interface. The 1:2 region accounts shows parametric instability of the parametrically driven sine-Gordon model (2).

oscillatory decorated interfaces. The colored region of Fig. 4 shows the area where one expects to find oscillating decorated interfaces from the amplitude equation (7). It is important to note that the bifurcation diagram obtained with the amplitude equation (7), close to the natural frequency $\left(\omega \sim \omega_{0}\right)$, is similar to that obtained from the parametrically driven sine-Gordon equation (2) (cf. Figs. 2 and 4). All the corrections ignored to obtain the amplitude equation modify the Arnold tongues [20] (see the analytic and numerical curves of Arnold tongues shown in Fig. 4).

The bifurcation diagram in Fig. 2 shows that for small forcing amplitude there is a transition from a flat to an oscillatory decorated interface. When increasing the forcing amplitude, the decorated interface becomes unstable by means of the asymptotic state undergoing a spatial instability. Indeed, one expects to find a rich morphology of decorated interfaces. Figure 5 shows an oscillating decorated interface between two waves. In vertically driven granular media, decorated interfaces

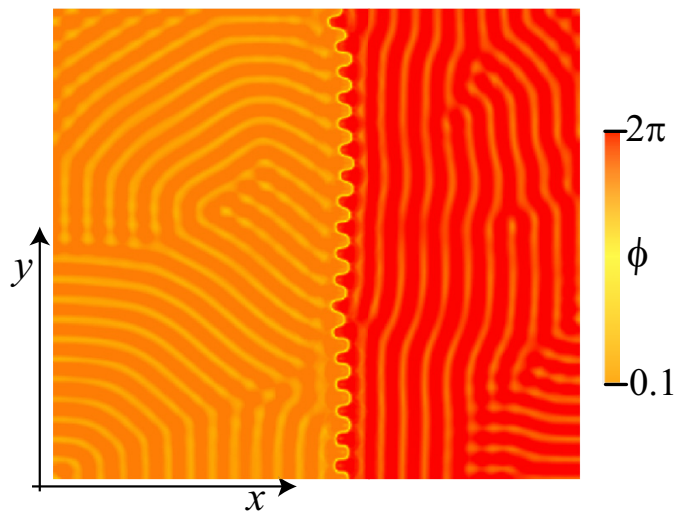

FIG. 5. Oscillating decorated interface of the parametrically driven sine-Gordon model (2) for $\omega_{0}=1.0, \beta=1.0, \omega=1.12$, $\gamma=1.46$, and $\mu=0.2$.

are observed between oscillating hexagonal patterns [10]. Understanding these interfaces is an open problem.

\section{CONCLUSIONS}

In conclusion, we have characterized the origin of the transition from a flat to an oscillating decorated interface in parametrically driven systems. This transition is a parametric resonance between the frequencies of interface modes and of the forcing. A magnetic plane under the influence of an oscillating magnetic field and an extended Josephson junction under the influence of an alternating current exhibit this transition. Likewise, this phenomenon has been observed in vertically driven granular media, however, there is not a satisfactory explanation in this context. An extension of the theory presented here for strongly dissipative systems could explain these observations.

\section{ACKNOWLEDGMENTS}

M.G.C. is grateful for financial support from FONDECYT through Project No. 1150507. E.B.-C. is grateful for financial support from CONICYT through Becas Magister Nacional, Contract No. 22151824. A.O.L. is grateful for financial support from JSPS KAKENHI through Grant No. 26103006 and CONICYT Becas Chile through Grant No. 74170017.
[1] G. Nicolis and I. Prigogine, Self-Organization in Nonequilibrium Systems (Wiley, New York, 1977).

[2] L. M. Pismen, Patterns and Interfaces in Dissipative Dynamics (Springer, Berlin, 2006).

[3] M. Cross and H. Greenside, Pattern Formation and Dynamics in Nonequilibrium Systems (Cambridge University Press, New York, 2009).

[4] J. S. Langer, Instabilities and pattern formation in crystal growth, Rev. Mod. Phys. 52, 1 (1980).

[5] T. Vachaspati, Kinks and Domain Walls: An Introduction to Classical and Quantum Solitons (Cambridge University Press, Cambridge, 2006).
[6] P. Coullet, J. Lega, B. Houchmandzadeh, and J. Lajzerowicz, Breaking Chirality in Nonequilibrium Systems, Phys. Rev. Lett. 65, 1352 (1990).

[7] M. G. Clerc, S. Coulibaly, and D. Laroze, Nonvariational Ising-Bloch transition in parametrically driven systems, Int. J. Bifurcat. Chaos 19, 2717 (2009).

[8] M. G. Clerc, S. Coulibaly, and D. Laroze, Localized states and non-variational Ising-Bloch transition of a parametrically driven easy-plane ferromagnetic wire, Physica D 239, 72 (2010).

[9] P. B. Umbanhowar, F. Melo, and H. L. Swinney, Periodic, aperiodic, and transient patterns in vibrated granular layers, Physica A 249, 1 (1998). 
[10] D. Blair, I. S. Aranson, G. W. Crabtree, V. Vinokur, L. S. Tsimring, and C. Josserand, Patterns in thin vibrated granular layers: Interfaces, hexagons, and superoscillons, Phys. Rev. E 61, 5600 (2000).

[11] A. Hubert and R. Schäfer, Magnetic Domains: The Analysis of Magnetic Microstructures (Springer Science + Business Media, Berlin, 2008).

[12] I. D. Mayergoyz, G. Bertotti, and C. Serpico, Nonlinear Magnetization Dynamics in Nanosystems (Elsevier, Oxford, 2009).

[13] J. C. Eilbeck, P. S. Lomdahl, O. H. Olsen, and M. R. Samuelsen, Comparison between one-dimensional and two-dimensional models for Josephson junctions of overlap type, J. Appl. Phys. 57, 861 (1985).

[14] H. J. Mikeska, Solitons in a one-dimensional magnet with an easy plane, J. Phys. C 11, L29 (1978).
[15] E. Berrios-Caro, M. G. Clerc, and A. O. Leon, Flaming $2 \pi-$ kinks in parametrically driven systems, Phys. Rev. E 94, 052217 (2016).

[16] J. Cuevas-Maraver, P. G. Kevrekidis, and F. Williams, The SineGordon Model and its Applications (Springer, Berlin, 2014).

[17] Y. S. Kivshar and B. A. Malomed, Dynamics of solitons in nearly integrable systems, Rev. Mod. Phys. 61, 763 (1989).

[18] L. D. Landau and E. M. Lifshitz, Mechanics, Course of Theoretical Physics Vol. 1 (Pergamon, Oxford, 1976).

[19] N. N. Bogolyubov and Y. A. Mitropolskii, Asymptotic Methods in the Theory of Nonlinear Oscillations (Gordon \& Breach, Delhi, 1961).

[20] M. G. Clerc, C. Fernández-Oto, and S. Coulibaly, Pinningdepinning transition of fronts between standing waves, Phys. Rev. E 87, 012901 (2013). 